\title{
EFFICIENCY AND EFFECTIVENESS APPROACHES IN SPATIAL DATA COLLECTION OF VRISA AFTER LESVOS EARTHQUAKE
}

\author{
D. Kavroudakis ${ }^{1,}{ }^{*}$, N. Soulakellis ${ }^{1}$, K. Topouzelis ${ }^{2}$, S. Chatzistamatis ${ }^{3}$, C. Vasilakos ${ }^{1}$, A. Papakostantinou ${ }^{1}$, G. Tataris ${ }^{1}$, C. \\ Kalloniatis $^{3}$, O. Rousou ${ }^{1}$, M. Batsaris ${ }^{1}$, K. Chaidas ${ }^{1}$, P. Kalaitzis ${ }^{1}$ \\ ${ }^{1}$ Department of Geography, University of the Aegean, Lesvos, Greece - dimitrisk@ aegean.gr \\ ${ }^{2}$ Department of Marine Sciences, University of the Aegean, Lesvos, Greece \\ ${ }^{3}$ Department of Cultural Technology and Communication, University of the Aegean, Lesvos, Greece
}

KEY WORDS: Efficiency, effectiveness, spatial data collection, spatial data management

\begin{abstract}
:
Efficient and effective spatial data collection is crucial in cases of catastrophic events. Resources efficiency and project effectiveness are two aspects that need special attention especially when there are spatial and temporal constraints. There is limited literature regarding efficiency and effectiveness in spatial data collection approaches. This work elaborates on the collection of spatially-aware data from a diverse scientific group of teams after the catastrophic earthquake of $\mathrm{Mw}=6.3$ in Vrisa village, Lesvos island in Greece in June 2017. More specific we deal with challenges faced by six teams of experts (Topographic team, House conditions data collection team, Unmanned Aerial Vehicle team, 3D Laser Scanner team, Photogrammetry team and Privacy team). The scientific teams had to collect accurate spatial data for the same area, during a period of 20 days after the earthquake. That was a challenging task due to restrictions in the area and complexity/diversity of spatial data. We present the methodological approaches followed for efficient and effective data-capturing, and we propose a framework of team/data management under concurrent data collection by scientific teams after catastrophic events such as earthquakes.
\end{abstract}

\section{INTRODUCTION}

Natural hazards such as earthquakes can have an enormous effect on populated places regarding both life-threating conditions and socio-economic impact. The east part of the Mediterranean basin is a very active tectonic area with an increased number of earthquake events.

Post-disaster activities from authorities require rapid approaches of handling a large amount of data. Understanding and quantifying the extent of a disaster may sometimes require a considerable amount of time especially if the geographical area affected is considered dangerous for emergency services to operate. More specific, after a catastrophic event such as an earthquake, there is a need for evaluation of the condition of human-made structures and services networks such as electricity and watering. That may be a time-consuming process which requires specialized personnel. Also, in cases where cultural and historic structures are also affected, there is a need for additional specialized teams to assess the damages and record the affected structures by advanced equipment. Finally, postdisaster services may also include the efficient evacuation of populated places and the management of high-value possessions of the affected individuals.

Post-disaster assessment actions require rapid data collection approaches and accurate measurements of affected structures. That may be a challenging task, especially when considering possible aftershocks, dangerous semi-collapsed structures and time-consuming tasks such as data validation. It is therefore essential considering efficient and effective data acquisition methods that will minimize collection time and maximize the accuracy of collected data.
Efficiency and effectiveness have been studied in many contexts over the last decades, especially in the scientific field of management. The work of Ostroff et al. (1993) examined the relationship between efficiency and effectiveness of organizations showing that behavioral aspects of involved personnel may be a significant contributory factor towards efficiency and effectiveness improvement of a project. The work of Frøkjær et al. (2000) regarding usability measuring the correlation between effectiveness and efficiency showed that there is no correlation between these two aspects of project management and that measuring them may be very subjective in some contexts. Furthermore, Jeong and Phillips (2001), presented a methodology for measuring overall equipment effectiveness based on 'product unit analysis' and 'state analysis' approaches, indicating that measuring efficiency and effectiveness may include very complex approaches of identifying outputs value (efficiency) and targeting optimization (effectiveness). Another notable work regarding efficiency and effectiveness is one of Mouzas (2006) which deals with the balancing act between these two terms in the context of business management. That study illustrates the importance of linking the two terms especially when dealing with project team performance. Finally, one of the most recent influential works about project efficiency and effectiveness is the work of Sundqvist et al. (2014) which evaluates recent literature in the field and concludes that there is not a single clear definition of both terms and that there is a need for clear definition of the terms in the relevant context of each scientific field.

This paper illustrates the work undertaken by a scientific team after the Vrisa earthquake in June 2017 (Lesvos island, Greece) in order to collect spatial data. After discussing the work 
undertaken by each of the six teams we illustrate efficiency and effectiveness characteristics of the work undertaken.

\section{BACKGROUND}

In line with the recent relevant literature in the field, this work defines efficiency of a scientific team as the ability to function in the best possible manner with the least waste of time and effort. Measuring efficiency is mainly based on the quantification of the relationship between inputs and outputs of a system. In other words, efficiency could be defined as the volume of resources used in a project over the final deliverables of the project. In the context of this work, efficiency is conceptualized as the relationship between capital used for the Vrisa Project (equipment, materials, time, costs) over the value of the scientific results produced by the team.

Effectiveness refers mainly to the capability of accomplishing a purpose and producing the intended result. In the context of project management, this is the capability of achieving a project's goal. Vrisa project effectiveness can be conceptualized as the capability of the team to achieve the desired outcome and produce the intended deliverables. Effectiveness is a measure of the output while efficiency is a measure of the relationship between input and output. The work of Hyväri (2006) illustrates in much detail the term of effectiveness in the context of project management. In the context of this work, we will illustrate the approaches followed by six scientific teams for the collection of spatial data after a natural disaster on an island in Greece.

\section{METHOD}

On $12^{\text {th }}$ of June 2017, an earthquake of Mw 6.3 occurred in the North-East part of the Aegean sea, close to Lesvos island. It affected most of the south part of the island with a single fatality in Vrisa village. This village was one of the most affected populated areas with an increased number of damages in human-made structures. Authorities required information regarding the general conditions of all human-made structures in the village in order to come up with relevant policies for the reimbursement of the owners. Entry to the village was prohibited for safety reasons and protection of exposed properties. There was a need for reliable data regarding the condition of all structures in the village which will form the basis for decision-making from Regional authorities. Thus, an interdisciplinary scientific team was assembled for the collection and analysis of spatial data in the affected village of Vrisa. The data collection process started on $13^{\text {th }}$ of June 2017 and lasted up until the $6^{\text {th }}$ of August 2017. The scientific teams were: 1) Topography team, 2) House conditions team, 3) UAV team, 4) 3D Laser Scanner team, 5) Photogrammetry team, and 6) Privacy team. Vrisa Project was essentially a rapid collection of accurate spatial data and the development of necessary deliverables for the damage assessment acts by the relevant authorities. This project is a very interesting case study of analyzing efficiency and effectiveness of spatial data collection approaches mainly due to rapid and concurrent data capturing needs. Access permit to the affected village was only granted for 30 days, and all six scientific teams had to work concurrently in the area avoiding overlapping.

\subsection{Efficiency and effectiveness in Vrisa Project}

In order to estimate the efficiency of the Vrisa Project we need to define a measure for quantification of this term. One approach is to quantify period over the value of the output (days/output value). Another metric could be the relationship between the cost of the project and the value of the output (project budget / output value). In the context of this work we use the second approach as a rough estimation of efficiency. The first part of the fraction is $€ 38,000$ gross which is essentially $€ 32,300$ net. The second part of the fraction is the value of the final products delivered from the University of the Aegean to the relevant stakeholders for further use in restoration and protection of the cultural heritage of the village. Vrisa is an officially a recognized protected-area in terms of cultural heritage. Thus, any act towards the preservation of its initial state and image contributes towards the protection of this heritage. In order to understand the value of the project's deliverables, we present the work undertaken by each of the six scientific teams.

\subsection{Scientific teams}

The aim of the topography team was to distribute and measure the Ground Control Points (GCPs) in Vrisa settlement. The purpose of GCPs network is to allow the necessary georeference of the aerial images acquired from the Unmanned Aircraft System (UAS) survey and also the georeference of the Laser Scanner's stations (laser team). The technique employed for the topographic survey is the Real Time Kinematic (RTK) were two GPS receivers are used. The first receiver was placed on a known point as a base-station transmitting the corrections to the rover receiver which is on the move measuring the coordinates of topographic points. Data collection was conducted during a week with the use of the Magellan ProMark3 RTK. The first day after the earthquake the topography team placed the base GPS receiver on the pedestal of the National Trigonometric Network (NTN) located at the top of the hill named 'Korona', $\mathrm{SW}$ of the settlement of Vrisa with known coordinates $\mathrm{X}=$ $689641.55 \mathrm{Y}=4322030.77$ and $\mathrm{Z}=135.98$. The next step was the installation of two new base-stations (BS) in the area of the settlement. First BS was placed in the courtyard of the Primary School, $1.2 \mathrm{~km}$ away from the base of the NTN, with a horizontal error of $0.7 \mathrm{~cm}$ and an altitude error of $1.1 \mathrm{~cm}$. Second BS was placed very close and south of the Metal Bell Tower, in front of the small church of the Cemetery of Vrisa at a distance of $1,4 \mathrm{~km}$ from the base of the NTN, with a $0.9 \mathrm{~cm}$ horizontal and $1.5 \mathrm{~cm}$ elevation errors respectively. These positions were chosen as they are at the two hills of the village and above all GCPs, in order to obtain more accurate measurements. Respectively, the rover receiver placed to those two positions and in static mode collected the coordinates. The next step was the distribution and the coordinates' collection of the GCPs. Every day, the base receiver was placed either on the school base or the church base, accordingly to the area of the placement of the GCPs. Thus, in RTK mode, 157 GCPs were placed uniformly in the whole area and with very high accuracy, more specifically, with an average horizontal error of $0.8 \mathrm{~cm}$ and an average elevation error of $1.3 \mathrm{~cm}$. All the coordinates of the GCPs collected in the Greek reference system GGRS87/Greek Grid (EPSG:2100) (Butler et al., 2018) and marked on the ground with a red spray or on metal screws. During the process, there were several problems with the collapse of buildings from the earthquake. Inability to sign the points because of lots of debris on the streets, but also difficulty in receiving a RTK signal due to the buildings of the settlement of Vrisa.

The aim of house conditions team was to accurately collect the house condition category (red, yellow, green) of all buildings in Vrisa village. Designated red houses should be demolished. Designated yellow structures will need interventions and the 
designated green structures are the ones that won't need any further improvements. Secondary target of this team was to collect any additional information regarding building's material and structure such as building category, building main material, roof type and age category. Data collection process took place in a single day (12 hours of surveying) recording 655 houses and capturing 110 images. We used hand-held tracking devices with Android Operating System, along with a customized software which was developed for this project. This 'app' includes an extended GUI specifically designed for capturing house conditions data. Our team was also equipped with high efficiency sun-power panel for the (unlimited) expansion of mobile power. The data collection process was conducted by two individuals, working alongside and capturing each of the 14 sectors in serial mode. Every 100 records of data, evaluation was conducted and then backed-up to a file-server (via 4G GSM network connection). The post-collection process of this team included: a) standardization of collected spatial data into groups, b) evaluation of the spatial accuracy of data, c) restructure topological errors due to GPS inaccuracy, d) association of qualitative information with spatial data. The final output of this team is a spatial point-dataset with extended information about house condition category (red, yellow, green) and attached qualitative information regarding the structure. That dataset is useful for emergency response teams dealing with compensation issues and surveying agencies need to evaluate the current overall affected houses in the area. Difficulties faced by this team include weather conditions and access to somehow dangerous spots in the area. Potential improvements of the followed data collection method, include the use of a web map for adding the data in real time. This may decrease the collection process time to less than 10 hours as we could have record multiple houses in less than 3 minutes time just by adding information on web-map with a pop-menu. In other words, instead of moving physically in front of each house in the village for the GPS device to collect the coordinates, we could have pass-by and assign the information on a web-map which already includes coordinates.

The UAS team conducted more than 40 survey flights using an Unnamed Aerial System to provide 3D geovisualizations of the Vrisa village. The main aim of the survey flights was two-fold. Firstly, a small part of the surveys was conducted to create subdecimeter spatial resolution orthophoto maps the day after the earthquake to help all relevant stakeholders (national and local organizations) to prioritize the needed actions thought the Vrisa settlement. Secondly, the most significant part of the data collected in the survey flights was the implementation of very detailed a 3D geovisualization of the settlement. During the survey flights, various combination of UAS and sensors were used to achieve a very high in resolution mapping and 3D visualization of the existing state of the settlement. The data acquisition was implemented at different heights with the use of five (5) cameras. The cameras used were a) a Sony A5100 camera with a fixed focal length of $19 \mathrm{~mm} \mathrm{~b}$ ) three (3) Canon IXUS 160 and c) a multi-spectral camera. Furthermore, for the creation of the 3D geovisualization of the settlement, a UAS equipped an innovative noncommercial multi-camera rig was used. The UAS team delivered the following end-products:

1. Orthophoto map and DSM (13 $3^{\text {th }}$ June 2017),

2. Orthophoto map and DSM (25 th July 2017),

3. Multi-spectral Orthophoto map $\left(20^{\text {th }}\right.$ June 2017),

4. High detailed three-dimensional representation (3D model) of the Vrisa settlement.

The use of UAS in combination with the Structure from Motion (SfM) method and multi-camera rig data provide very high- resolution images, capturing the morphology of the entire Vrisa settlement few days after the earthquake. The 3D models of the damaged buildings produced from UAS-SfM can depict in high detail and in the most efficient way all the results of the destructive earthquake of the settlement buildings.

Photogrammetry team, conducted data collection in 6 days with 2-3 sectors per day collecting more than 20000 images. The team gathered data with two DSLR cameras. Two persons acquired photographs and a third person was located in a specific location with all the other equipment and belongings, collecting the data from the memory cards to an external hard disk through a laptop. The field equipment was two NIKON D3400 DSLR cameras, two memory cards, four batteries, two laptops and two external hard disks 1 TB each. Two more surveys were needed in order to collect scale measurements and acquire photographs from specific sections where initial photoalignment was not adequate. Each DSLR user was surveying different road sections while both users were working at the same sector.

Initial data evaluation was conducted every 3 road sections, during data transfer from cameras to laptop PCs. The second evaluation was conducted daily when uploading data at the base station in the University lab facilities. Data backup was taking place daily in both external hard disk drives and a file server physically located at the premises of the University lab. The aim of the analysis was to create 3D models of all buildings in Vrisa village photogrammetrically. The team produced three final products for each house in the area: a) a series of photographs documenting house damages, b) a 3D model (as a point cloud) of the building, c) 3D embedded PDF files of each building. Final products of this team, aid agencies compensating damaged-house owners to estimate the volume of buildings. Additionally, can be used by engineering scientists studying the type and format of damages as well as architects approaching new architectural designs based on initial house structures. Challenges during data collection include difficulties accessing some human-made structures due to high density of debris. Also, due to many aftershocks in the area, it was difficult to access neighborhoods with half-demolished structures on safety grounds. Data collection method can be improved by using more scale targets and extra photographs from UAS low altitude flights for possible fusion with terrestrial images.

The aim of the laser scanning team was to provide a highresolution 3D post-earthquake visualization of Vrisa settlement. The survey was conducted using the Terrestrial Laser Scanner (TLS) FARO Focus ${ }^{3 \mathrm{D}}$ (Chow et al., 2012), which is based on the phase-shift measurement principle. The fieldwork was undertaken in thirteen days, where the first two days the team planned the scanning survey, on the basis of the coverage of the largest possible area, e.g. both damaged and undamaged buildings. The team acquired a total number of 162 scans in eleven days, with an average number of 15 scans per field work day. The team consisted of three individuals, working alongside. Two members of the team operated the laser scanner and the third member of the team (team manager) was responsible for data management, backup, evaluation, and quality control of the data. During each scan the following data were collected: a) a dense point cloud of $360^{\circ} \times 305^{\circ}$ field-ofview and spatial resolution less than $1.5 \mathrm{~cm}, \mathrm{~b}$ ) digital compass measurements, c) inclinometer measurements, d) pictures from the integrated digital camera of the scanner, e) control distances of the scanned area measured by measurement-tape on fixed 
objects such as doors and windows. The scanning process was undertaken on positions where their coordinates were premeasured in the Greek Geographical reference system GGRS87/Greek Grid (EPSG:2100) (Butler et al., 2018) with Real Time Kinematics (from Team 1: Topography team). After each scan, a single laptop computer was used for data backup and evaluation using local WiFi connection between the 3D scanner and the computer. During the survey, we had to take into account inhibitory factors such as the avoidance of use of locations where physical integrity of team members could be compromised, as well as the limitation of avoiding scanning from privately owned premises such as house courtyards. Also, due to weather conditions, team members were exposed to relatively high temperatures for prolonged periods of time. Data evaluation consisted of visual inspection of the scanned area point cloud (for undesired reflections and moving objects such as cars or individuals) as well as the comparison of scanned distances with the manually measured ones. Scans that did not meet all the above criteria were rejected and acquired again. Finally, a daily backup was undertaken to an in-house file server.

The post-processing work followed the field work and it was undertaken in sixteen days. As mentioned above, the raw data from a laser scan is a dense point cloud, mapping in 3D the scanned area. During scanning, however, the 3D laser scanner captures points that may not necessarily be used such as vegetation. Also, the points are affected by a disorder generally referred to as "noise" that depends on the object's surface characteristics. Thus, for each scan, the point clouds were processed: a) cleaned from noise and undesired areas, and b) applied a texture using the acquired photos. Finally, the last evaluation and quality control took place. The final deliverables of this team were a map with georeferenced scanner positions and individual dense point clouds with texture acquired from these positions.

Potential improvement of the data collection approach is.the use of a second TLS in order to decrease the number of days needed for fieldwork. The deliverables of this team can be used from all stakeholders that are responsible of the restoration and reconstruction such as engineers who can identify the damages accurately. Also, the deliverables can be used for further research on the structural impacts of an earthquake on buildings.

Privacy team, collected the intermediate products of photogrammetry team and 3D laser scanner team, in order to check for privacy issues in the deliverables. The focus of this team was to identify and correct privacy issues in digital images and 3D scans and take any possible step towards privacy protection of individuals and their properties. An exhaustive processing approach was undertaken which included supervised tagging of images and 'blurring' of potentially risky spots. This time-consuming approach required a considerable amount of hard-disk storage space.

\section{DISCUSSION AND CONCLUSION}

The deliverables of the above scientific teams are diverse and include a wide range of products from maps to 3D models and special type of 3D-PDF files as well as topographic records and orthophoto maps. These products are helpful for engineering activities in the area as they include accurate measurements and representations of all buildings in the village. The total value of these products is well beyond the range of the budget of the Vrisa project.

The results of this project can help relevant agencies crosscheck the details of buildings before compensating the residents of the village. In other words, the outputs of this project will reduce the estimated time of inspections from the relevant agencies.

A rough estimation of efficiency of this project can be made based on the contribution of the results to each engineering project in the village. For a $20 \%$ contribution and with almost 400 engineering projects in the village (of $€ 1,000$ mean cost per project) we can conclude that there is an average gain of $€ 80,000$ for all engineering projects that will take place in Vrisa village. Comparing this estimation with the cost of Vrisa project $(€ 30,000)$ we can conclude that it was a very efficient project with a valuable outcome that will play an important role in most of the engineering projects of the village in the future.

An additional outcome of this project is the development of innovative methods from the scientific teams. Some new approaches of fast spatial data collection have been developed from all six teams. These novel approaches have been developed on the basis of reducing data collection time, combination of data sources and accuracy reduction approaches. The Vrisa project was both efficient ('doing things right') and effective ('doing the right things') and will form the basis for a number of future scientific research projects in the field.

\section{ACKNOWLEDGEMENTS}

This paper is a result of the research project " $3 \mathrm{D}$ mapping of Vrisa settlement after the 12th June Lesvos earthquake" funded by the North Aegean Region. The authors would like to thank Professor Pavlogeorgatos G., Doukari M., Drolias A., Mauroeidi A., Zorbas K., Papazis N., Moustakas, A. and Makri D. for supporting the processing stage of this project.

\section{REFERENCES}

Butler, H., Schmidt, C., Springmeyer, D., and Livni, J., 2018. GGRS87 / Greek Grid: EPSG Projection -- Spatial Reference [WWW Document]. URL

http://spatialreference.org/ref/epsg/ggrs87-greek-grid/ (accessed 2.11.18).

Chow, J., Lichti, D., and Teskey, W., 2012. Accuracy assessment of the Faro Focus3D and Leica HDS6100 panoramic type terrestrial laser scanner through point-based and plane-based user self-calibration. In: Proceedings of the FIG Working Week: Knowing to Manage the Territory, Protect the Environment, Evaluate the Cultural Heritage, Rome, Italy.

Frøkjær, E., Hertzum, M., and Hornbæk, K., 2000. Measuring Usability: Are Effectiveness, Efficiency, and Satisfaction Really Correlated? In: Proceedings of the SIGCHI Conference on Human Factors in Computing Systems, CHI '00. ACM, New York, NY, USA, pp. 345-352.

Hyväri, I., 2006. Project management effectiveness in projectoriented business organizations. International journal of project management, 24(3), pp. 216-225.

Jeong, K.Y. and Phillips, D.T., 2001. Operational efficiency and effectiveness measurement. International Journal of Operations \& Production Management, 21(11), pp. 1404-1416. 
Mouzas, S., 2006. Efficiency versus effectiveness in business networks. Journal of Business Research, 59(10-11), pp. 11241132 .

Ostroff, C. and Schmitt, N., 1993. Configurations of Organizational Effectiveness and Efficiency. The Academy of Management Journal, 36(6), pp. 1345-1361.

Sundqvist, E., Backlund, F., and Chronéer, D., 2014. What is Project Efficiency and Effectiveness? Procedia - Social and Behavioral Sciences, 119, pp. 278-287. 